\title{
Maintenance metronomic chemotherapy combined with conventional treatment for metastatic breast cancer patients
}

\author{
Sherif Elzawawy', Gehan Khedr', Basma Elsabaa² \\ Departments of ${ }^{1}$ Clinical Oncology and ${ }^{2}$ Pathology, Faculty of Medicine, Alexandria University, Alexandria, Egypt
}

Purpose: To evaluate the progression free survival, response rate and toxicity for patients who were treated with conventional therapy combined with metronomic chemotherapy.

Methods: patients received either chemotherapy followed by maintenance metronomic chemotherapy with or without hormonal therapy or primarily treated with hormonal therapy concomitant with metronomic chemotherapy. Metronomic chemotherapy consists of cyclophosphamide $50 \mathrm{mg}$ tablet daily and methotrexate $2.5 \mathrm{mg}$ twice daily on days 2 and 5 weekly, continued until disease progression or development of unacceptable toxicity.

Results: after median follow up of 18 months, 40 patients were assessed. The progression free survival was $52.5 \%$ and the median time to disease progression was 10.4 months. The overall clinical benefit (complete response [CR]+partial remission [PR]+stable disease [SD]) was $52.5 \%$ with no G3 or G4 toxicities encountered for metronomic therapy. The median time to disease progression was 12.5 months for estrogen receptor (ER)+ve, human epidermal growth factor receptor 2 (Her2)-ve (progression free survival [PFS]: 67.5\%) compared to 8 months for ER+ve, Her2+ve (PFS: 10\%), 9 months for ER-ve, Her2+ve (PFS: 10\%) and 8 months for triple negative (PFS: 12.5\%). The difference was statistically significant $(P=0.018)$.

Conclusion: maintenance metronomic cyclophosphamide and methotrexate demonstrated efficacy and provided durable disease stabilization especially for ER positive patients. The low costs and minimal toxicity allow patients to continue treatment for several months and support its use as an additional therapeutic tool.

Keywords: Maintenance metronomic, Metastatic breast cancer

\section{INTRODUCTION}

The treatment duration with chemotherapy in metastatic breast cancer patients remains a major issue, whether or not to continue chemotherapy until disease progression or development of unacceptable toxicity [1].

There is no overall survival benefit with prolonged use of continuous chemotherapy versus shorter course of chemotherapy but some trials showed superior response rate and time to disease pro-

Received: Aug 28, 2015 Accepted: Oct 26, 2015

Correspondence to: Sherif Elzawawy

Department of Clinical Oncology, Faculty of Medicine, Alexandria

University, 456 El Horia Street, Roushdy, Alexandria, Egypt

Tel: +20-2-01223757606 Fax: +03-486-2506

E-mail: sherifzawawy@yahoo.com

Copyright $@$ Korean Society of Surgical Oncology

This is an Open Access article distributed under the terms of the Creative Commons Attribution Non-Commercial License (http://creativecommons.org/licenses/by-nc/3.0) which permits unrestricted non-commercial use, distribution, and reproduction in any medium, provided the original work is properly cited. gression [2]. So, the use of prolonged versus shorter chemotherapy needs to be weighed against the detrimental effects of continuous chemotherapy on overall quality of life [3].

Metronomic chemotherapy refers to the frequent, even daily, administration of chemotherapeutics at doses significantly less than the maximum tolerated dose (MTD), with no prolonged drug-free breaks [4]. While the main target of chemotherapeutic drugs administered at the maximum tolerable dose is the tumor cells, the main target for metronomic agents, is the tumour endothelium.

Metronomic treatment is more effective on tumor endothelial cells than the conventional large single bolus doses [5]. This is because tumoral vascular endothelial cells, in contrast to the endothelium of normal mature blood vessels proliferate rapidly and are vulnerable to cytotoxic agents. However, the long interval between cycles of conventional chemotherapy permits the survival and regrowth of a small number of endothelial cells, allowing tumor angiogenesis to persist [6].

The mechanisms responsible for the anti-angiogenic activity of 
metronomic chemotherapy seems to be the induction of increased plasmatic levels of thrombospondin 1 that is a potent endothelial angiogenic inhibitor [7]. In addition, the tumor vasculature also depends on vasculogenesis mediated by circulating endothelial precursor cells (CEPs) originating from the bone marrow. The metronomic administration of cytotoxic drugs may inhibit the CEP release from the bone marrow [8].

Angiogenesis, however, is not the only target of metronomic chemotherapy since this treatment modality has also an immunomodulatory role. In particular, it inhibits a specific subpopulation of CD4+, CD25+ regulatory T cells (Treg) which suppress the activity of both tumor-specific (CD8+ cytotoxic T lymphocytes and CD4+ T helper cells) and tumor-unspecific effector cells (natural killer and nK T cells). Interestingly, Treg cells have been shown to be frequently increased in human cancers $[9,10]$.

The first trial to assess the efficacy of metronomic chemotherapy for metastatic breast cancer was reported by Colleoni et al. [11]. In this phase II study using daily oral cyclophosphamide and twiceweekly methotrexate, the overall response rate was 19\% (95\% confidence interval [Cl], $10.2 \%$ to $30.9 \%$ ) with an overall clinical benefit (complete response $[\mathrm{CR}]+$ partial remission $[\mathrm{PR}]+$ stable disease [SD]) of $31.7 \%$ and causing minimal toxicity. Plasma vascular endothelial growth factor (VEGF) levels decreased in all patients on therapy for at least 2 months.

Another trial observed that the administration of metronomic oral cyclophosphamide in association with letrozole as neoadjuvant approach is more efficacious than letrozole alone in elderly breast cancer patients and significantly reduced the intratumor expression of Treg [12]. Treg number at residual histology was inversely related with response $(P<0.03)$, and a greater Treg reduction was observed in responding patients $(P<0.03)$. This suggests that metronomic chemotherapy plus aromatase inhibitors may have an indirect antitumor mechanism of action through reducing Treg in breast tumors.

Also, Trastuzumab in combination with metronomic chemotherapy (MTX $2.5 \mathrm{mg}$, bid on day 1 and day 4 every week) and cyclophosphamide (CTX, 50 mg daily) was evaluated in metastatic breast cancer. There were 4 PR (18\%; 95\% Cl, 5\%-40\%), 10 SD (46\%), and 8 progressive disease (PD) (36\%). The overall clinical benefit was $46 \%$ and $27 \%$ for patients resistant to trustuzumab [13].

Based on these previous data, the present study was conducted to evaluate the efficacy of maintenance metronomic chemotherapy in combination with conventional systemic therapy as first line treatment for metastatic breast cancer patients.

\section{Aim}

The primary objective of this study was to evaluate the progression free survival for combined MTD chemotherapy followed by metro- nomic chemotherapy or concurrent hormonal and metronomic chemotherapy in metastatic breast cancer patients. The secondary objective was to evaluate for the response rate and adverse events.

\section{METHODS}

\section{Inclusion criteria}

Patients aged $\geq 18$ and $\leq 70$ years, pre- or postmenopausal, histologically confirmed metastatic breast carcinomas were included. Patients were either pretreated or not with chemotherapy and/or hormonal therapy in the adjuvant setting. All patients were candidate for first line treatment for their metastatic disease.

Hormone receptors and Her2/neu positive or negative patients were included. Patients had to have Eastern Cooperative Oncology Group (ECOG) performance status $\leq 2$, at least one measurable lesion or lytic bone lesions in absence of measurable disease. Adequate bone marrow reserve defined as white blood cells $>4,000 \mathrm{~mm}^{3}$, hemoglobin $>10 \mathrm{~g} / \mathrm{dL}$ and platelets $>100,000 \mathrm{~mm}^{3}$, adequate renal (serum creatinine $<1.2 \mathrm{mg} / \mathrm{dL}$ ) and hepatic functions (biliribin $<2$ $\mathrm{mg} / \mathrm{dL}$ liver enzymes $<2 \times$ upper limit of normal) are required. Written consents were taken from all patients and approval of the Ethical Committee was obtained.

\section{Exclusion criteria}

Patients with central nervous system metastasis or PD after 3 months of starting treatment or previous treatment by chemotherapy or hormonal therapy for metastatic disease.

\section{Study design}

All patients treated with either MTD chemotherapy for their metastatic disease (first line) and then received metronomic chemotherapy with or without hormonal therapy or primarily treated with hormonal therapy concomitant with metronomic chemotherapy.

Metronomic chemotherapy consists of cyclophosphamide $50 \mathrm{mg}$ tablet daily and methotrexate $2.5 \mathrm{mg}$ twice daily on days 2 and 5 every week. Treatment continued until disease progression or development of unacceptable toxicity.

\section{Efficacy and safety assessment}

All patients were subjected to base line complete physical examination, complete laboratory investigations including complete blood count, urea, creatinine, serum glutamic oxaloacetic transaminase, serum glutamic pyruvic transaminase, bilirubin, alkaline phosphtase, serum calcium, computed tomography (CT) scan chest, abdomen and pelvis and bone scan. Abnormal bone scans were assessed by X-ray or CT scan with bone window.

Tumor assessment included physical examination and laboratory 
investigations every month, CT scan chest, abdomen and pelvis every 3 months, bone scan repeated every 6 months unless abnormalities during physical examination or in laboratory investigations.

PFS which is the primary end point was defined as the time from the start of MTD chemotherapy or hormonal treatment to the time of first documentation of disease progression (increase by $25 \%$ or more) either clinically or radiologically or appearance of new lesions.

The secondary objective was to evaluate for the response rate evaluated by Response Evaluation Criteria in Solid Tumors guidelines. Adverse events were monitored continuously throughout the study and graded according to the National Cancer Institute Common Terminology Criteria for Adverse Events.

Metronomic treatment was withheld and delayed for 1 week in case of a neutrophil count $<1,000 \mathrm{~mm}^{3}$ and/or platelet count $<75,000 \mathrm{~mm}^{3}$. A 50\% dose reduction in the total amount of drug administered in each cycle was prescribed after hematological recovery. In case of a neutrophil count $<1,500$ but $>1,000 \mathrm{~mm}^{3}$ and/ or platelet count $<100,000$ but $>75,000 \mathrm{~mm}^{3}$, therapy was administered with a 50\% dose reduction in the total amount of drug administered in each cycle. Re-escalation of drug doses was attempted if close monitoring was possible. In the event of grade $\geq 2$ anorexia, nausea, vomiting, diarrhea, stomatitis, dryness of the mouth, epigastric pain or increase in transaminases, all therapy was postponed until symptoms subsided. A 50\% reduction of cyclophosphamide/ methotrexate (CM) was performed for the next cycle, with subsequent re-escalation to full dosage if tolerated.

\section{RESULTS}

From August 2012 through September 2014, a total of 42 patients were enrolled. The data cutoff point was in June 2015. Two patients were excluded due to disease progression at 3 months assessment. The clinical and pathological characteristics of these patients are presented in Table 1.

\section{Efficacy}

Three groups of patients were identified: (1) 8 patients received hormonal and metronomic therapy. (2) 9 patients received MTD chemotherapy followed by metronomic treatment. (3) 23 patients received MTD chemotherapy followed by metronomic and hormonal treatment.

At the end of MTD chemotherapy, 2/32 patients achieved complete response, 17/32 patients had partial response, and 13/32 patients with stable disease. All of those patients started metronomic chemotherapy according to the study design.

The assessment for patients treated with hormonal plus concurrent metronomic chemotherapy at 6 months showed, none of the 8 patients achieved complete response, 5/8 patients had partial response and $3 / 8$ patients remained with stable disease.

Overall assessment of all patients groups at 6 months: $2 / 40$ patients (5\%) achieved CR, 22/40 (55\%) had PR and 16/40 patients (40\%) had SD. At 9 months, 10 patients progressed, 2/40 patients (5\%) remained in $C R, 16 / 40$ patients (40\%) with $P R$, and $12 / 40$ (30\%) patients had SD.

At 12 months, 17 patients had progressed with 1/40 (2.5\%) stayed with $C R, 13 / 40$ (32.5\%) patients had PR and 9/40 patients

Table 1. Patients' clinical and pathological characteristics at baseline

\begin{tabular}{|c|c|}
\hline \multirow{2}{*}{ Characteristic } & $n=40$ \\
\hline & No. (\%) \\
\hline \multicolumn{2}{|l|}{ Age } \\
\hline$\leq 50 \mathrm{yr}$ & $21(52.5)$ \\
\hline$>50 \mathrm{yr}$ & $19(47.5)$ \\
\hline \multicolumn{2}{|l|}{ ECOG performance status } \\
\hline 0 & $19(47.5)$ \\
\hline 1 & $13(32.5)$ \\
\hline 2 & $8(20.0)$ \\
\hline \multicolumn{2}{|l|}{ Prior disease free duration } \\
\hline$\leq 1 \mathrm{yr}$ & $7(17.5)$ \\
\hline$>1 \mathrm{yr}$ & $10(25.0)$ \\
\hline Metastatic at presentation & $23(57.5)$ \\
\hline \multicolumn{2}{|l|}{ Prior adjuvant therapy } \\
\hline Chemotherapy & $4(10.0)$ \\
\hline Hormonal +/- chemotherapy & $13(32.5)$ \\
\hline \multicolumn{2}{|c|}{ No. of metastasis at time of study entry } \\
\hline Solitary & $4(10.0)$ \\
\hline Multiple & $36(90.0)$ \\
\hline \multicolumn{2}{|l|}{ Site of metastasis } \\
\hline Visceral & $18(45.0)$ \\
\hline Bone & $8(20.0)$ \\
\hline Visceral and bone & $13(32.5)$ \\
\hline Subcutaneous noduls & $1(2.5)$ \\
\hline \multicolumn{2}{|l|}{ Hormone receptors and Her2 status } \\
\hline ER, PR+ve, Her2/neu-ve & $27(67.5)$ \\
\hline ER, PR+ve, Her2/neu+ve & $4(10.0)$ \\
\hline ER, PR-ve and Her2+ve & $4(10.0)$ \\
\hline Triple-ve & $5(12.5)$ \\
\hline \multicolumn{2}{|c|}{ Systemic treatment for metastatic disease } \\
\hline Chemotherapy & $9(22.5)$ \\
\hline Hormonal & $8(20.0)$ \\
\hline Chemotherapy and hormonal & $23(57.5)$ \\
\hline \multicolumn{2}{|l|}{ No. of chemotherapy cycles } \\
\hline 6 Cycles & $29(72.5)$ \\
\hline 8 Cycles & $3(7.5)$ \\
\hline
\end{tabular}

ECOG, Eastern Cooperative Oncology Group; Her2, human epidermal growth factor receptor 2; ER, estrogen receptor; PR, partial remission. 
(22.5\%) with SD. Fifty months assessment showed no disease progression.

After a median follow up duration of 18 months (range, 9-26 months), 1/40 patient (2.5\%) sustained with $C R, 12 / 40$ patients $(30 \%)$ remained with $\mathrm{PR}$, and $8 / 40$ stable disease (20\%) with overall clinical benefit $(\mathrm{CR}+\mathrm{PR}+\mathrm{SD})$ of $52.5 \%$. Disease progression was observed in 19/40 patients (47.5\%). The progression free survival was $52.5 \%$ and the median time to disease progression was 10.4 months (Fig. 1).

Subgroup analysis according to ER and Her 2/neu status, revealed that $8 / 27(29.6 \%)$ patients ER positive, Her2-ve had progressed compared to $4 / 4$ (100\%) with ER+ve, Her2/neu+ve patients, 4/4 (100\%) with ER-ve, Her2/neu+ve, and 3/5 patients (60\%) with triple negative disease. The median time to disease progression was 12.5 months for ER+ve, Her2-ve (PFS: 67.5\%) compared to 8 months for ER+ve, Her2+ve (PFS: 10\%), 9 months for ER-ve, Her2+ve (PFS: 10\%) and 8 months for triple negative (PFS: 12.5\%). The difference is statistically significant $(P=0.018)$ (Fig. 2).

Analysis according to the disease free duration showed that, 6/7 patients (85.7\%) with disease free duration less than one year had

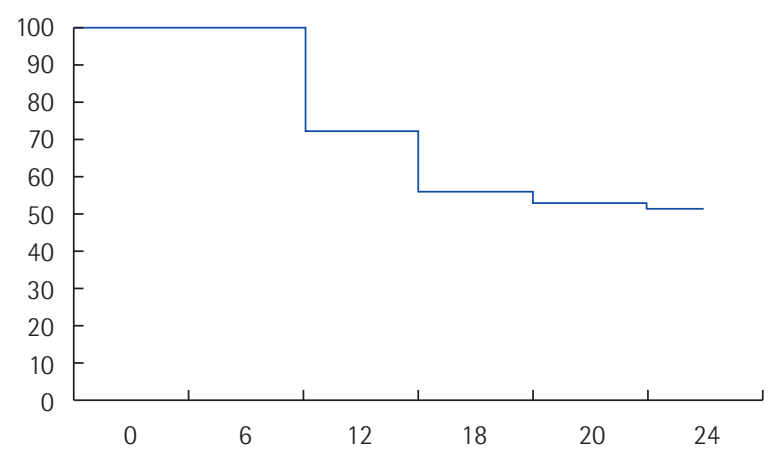

Fig. 1. Distribution of the studied patients regarding the progression free survival.

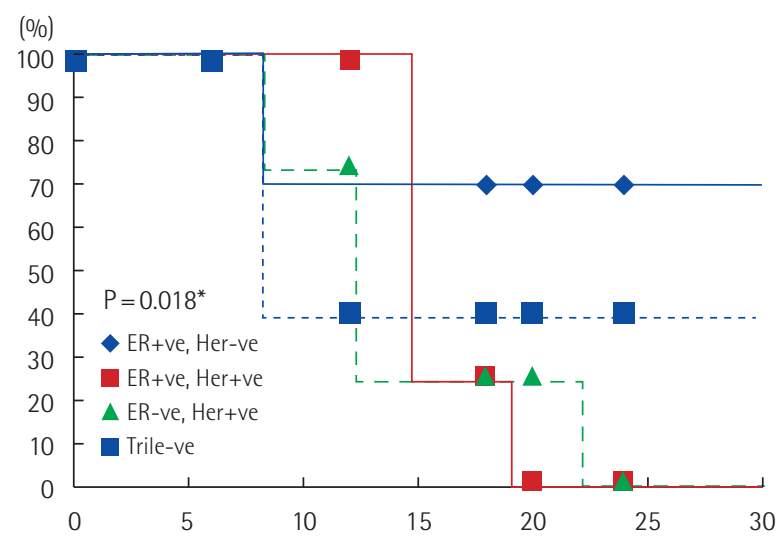

Fig. 2. Subgroup analyses according to ER and Her2/neu status. Her2, human epidermal growth factor receptor 2; ER, estrogen receptor. progressed compared to 4/10 patients (40\%) with disease free duration more than one year had progressed compared to $9 / 23$ patients (47.8\%) presented with stage IV had progressed. The median time to disease progression was 9 months (PFS: 14.3\%) for patients with disease free duration < 1 year compared to 10.5 months (PFS: 63.6\%) for patients with disease free duration $>1$ year and 10 months (PFS: 59.1\%) for those with stage IV at presentation. The difference is not statistically significant $(P=0.092)$ (Fig. 3).

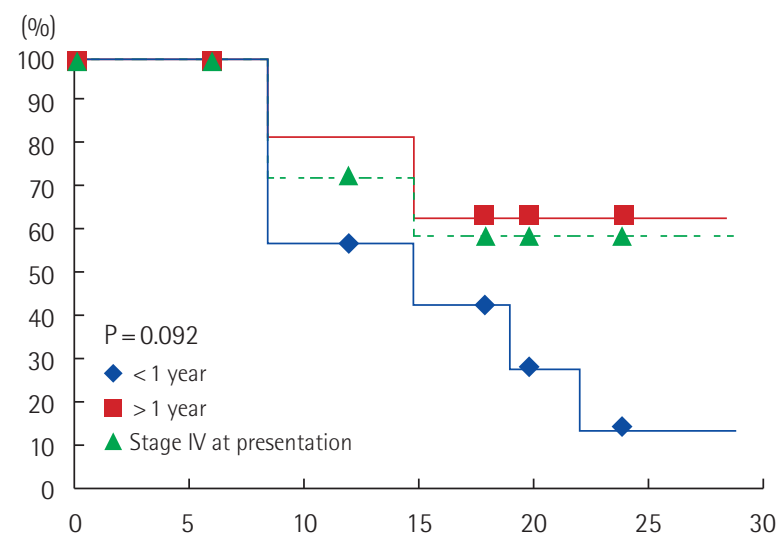

Fig. 3. Analysis according to the disease free duration.

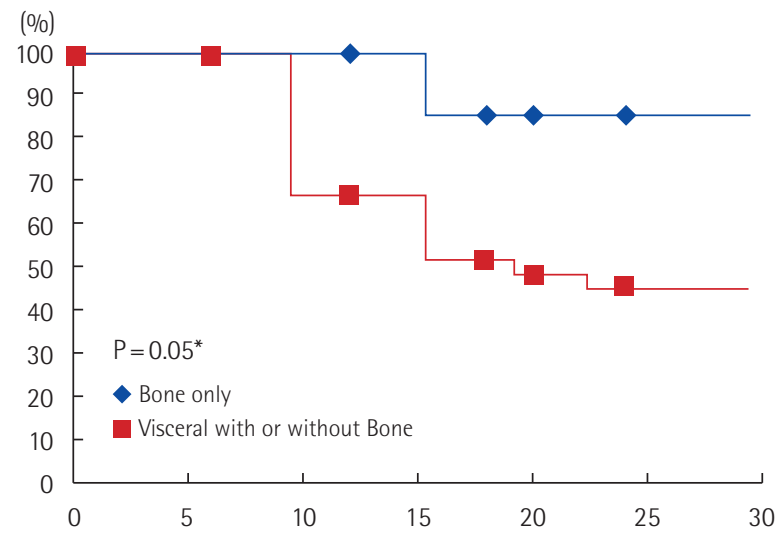

Fig. 4. Analysis according to the site of metastatic disease.

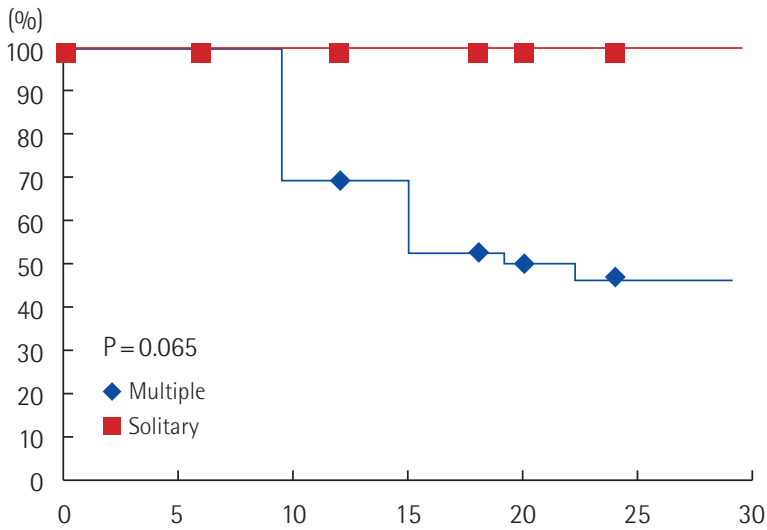

Fig. 5. Analysis regarding the number of metastatic sites. 
According to the site of metastatic disease, 1/8 patient (12.5\%) with bone only metastasis had progressed (PFS: 87.5\%) compared to $18 / 31$ patients $(58 \%)$ with visceral $+/$ - bone metastasis developed progression (PFS: 45.4\%). The difference is statistically significant $(P=0.05)$ (Fig. 4).

Regarding the number of metastatic sites, all patients (19/36) who developed progression had multiple metastatic sites (PFS: 47.2\%) compared to 0/4 (0\%) patients with solitary metastasis (PFS: 100\%). The difference is not statistically significant (Fig. 5).

Multivariate analysis was used to assess the prognostic value of various parameters (site of metastasis, number of metastasis, ER, Her2neu status, stage at presentation and disease free duration after adjuvant treatment) on outcome in term of PFS. It was found that ER-ve, Her2+ve and the presence of visceral metastasis are the most significant negative prognostic factors for time to disease progression. Patients with bone only metastasis and ER+ve, HER2 neu-ve have the best PFS whereas Patients with ER-ve, Her+ve combined with visceral metastasis have the worst outcome.

\section{Treatment toxicity}

During metronomic therapy, 2 patients discontinued metronomic treatment, one patient stopped metronomic therapy after one year due to intolerable gastritis and the other patient withheld treatment for 2 weeks due to grade 3 increased transaminases and resume treatment with $50 \%$ dose reduction.

Grade 1 anemia in 4 patients (10\%), 15 patients developed G1 neutropenia and 10 patients had G2 neutropenia, without febrile complications. Regarding gastrointestinal adverse events, only G1/2 nausea and dyspepsia observed in 18/30 patients (60\%) treated medically without treatment interruption. No alopecia was reported.

\section{DISCUSSION}

The concept of metronomicaly delivered therapy has become relevant for the treatment of cancer. It was originally developed to overcome drug resistance by shifting the therapeutic target from tumor cells to the tumor vasculature and therefore cytotoxic agent may result in beneficial antitumor effects even in tumors resistant to MTD regimens of the same drug [14]. An antiangiogenic activity is prominent with the protracted exposure to low doses of chemotherapeutics, if compared with their cyclic administration at the MTD [6].

Recent publications showed in vitro activity of taxanes and vinca alkaloids at chronic, low-dose exposure, which resulted in inhibition of blood vessel formation and growth. Fluorouracil administered continuously at low doses, which was reported to be successful for treating patients with breast carcinoma, appears to be as the first example of a metronomic schedule. Also, weekly paclitaxel appeared to be more active than standard 3-weekly administration of the drug [15-17].

Unlike studies evaluating the activity of classic cytotoxic agents, where shrinkage in tumor size is the objective, the absence of disease progression assumes a great importance as an end point in clinical trials using metronomic chemotherapy.

The largest clinical trials investigating metronomic chemotherapy efficacy have been conducted in patients with advanced breast cancer. The average response rates and overall clinical benefit reported in these studies were 39\% (range, 12\%-88\%) and 57\% (range, 24\%-93\%), respectively.

Comparison between different clinical trials using metronomic chemotherapy is quite difficult due to the small number of patients in each trial, the difference in study design, different inclusion and exclusion criteria, and the use of targeted therapy. None of these trials use metronomic therapy as a maintenance treatment after conventional systemic treatment as the present study.

The current trial is the first clinical experience reported of maintenance metronomic chemotherapy after conventional doses for patients with advanced breast cancer.

The first work using metronomic chemotherapy in a clinical setting was published in 2002 [11]. In which Colleoni et al. [11] evaluated the clinical efficacy of low-dose methotrexate and cyclophosphamide in heavily pretreated breast cancer patients, obtaining significant efficacy with minimal toxicity. Tumor regression was observed in 19\% of patients with an overall clinical benefit of 31.7\% (which is much lower than that in our study) (overall clinical benefit is $52.5 \%$ ). This lower outcomes compared to the present study (overall clinical benefit is $52.5 \%$ ) could be attributed to the difference in the study design, in the former study, metronomic treatment was administered as a third or fourth line treatment after progression on several lines of conventional chemotherapy in contrast to our study in which metronomic therapy was administered following first line conventional chemotherapy for their metastatic disease and also in responding patients.

Her2+ve patients in the present trial did not receive trastuzumab due to its cost. In Orlando et al. [13] study, Trastuzumab in combination with metronomic chemotherapy (MTX $2.5 \mathrm{mg}$, bid on day 1 and day 4 every week) and CTX (50 mg daily) was evaluated in metastatic disease (14 patients had PD at study entry and 11 had PD during previous trastuzumab therapy). There were 4 PR (18\%; 95\% Cl, 5\%-40\%), 10 SD (46\%), and 8 PD (36\%). The overall clinical benefit was $46 \%$ for patients who did not receive trustuzumab before and $27 \%$ for patients resistant to trustuzumab.

The identification of patients who might benefit from metronomic therapy is crucial for the optimization of the treatment strategy. 
In the present study, we found that hormone receptor status was significantly related to clinical benefit. The time to disease progression is statistically higher for ER positive patients compared to Her2 positive and triple negative patients (13.2 vs. 7.6 vs. 8.4 months). These results are in line with previously reported data in metastatic breast cancer, in which endocrine responsiveness was related to better PFS and overall survival. These results might be related to the biology of endocrine-responsive disease, which is characterized by indolent, low-proliferating tumors and, therefore, more likely to have prolonged stabilization $[18,19]$.

Moreover, there is a biologic rationale for improved activity of antiangiogenic treatment in endocrine-responsive tumors. Several growth factors influence proliferation and survival of ER-positive hormone resistant disease, in particular, VEGF is elevated in patients with endocrine-responsive disease who do not respond to hormone therapy, therefore contributing to disease progression and resistance to endocrine therapies [20].

No validated biomarkers currently exist for appropriately selecting patients with cancer for metronomic therapy, VEGF level, circulating endothelial cells and progenitor endothelial cells could be potent predictive tools for patient stratification and treatment monitoring, but the best methods of identification and measurement need to be determined.

Although, retrospective comparison of the present study results with the published data using chemotherapy and/or hormonal treatment alone without metronomic therapy for metastatic breast cancer patients showed no significant difference in the progression free survival or time to disease progression which is the primary end point, a larger study population is still required for proper evaluation of the results.

An important issue in this study, especially in the developing countries, is the treatment cost. In this prospect, the monthly cost of metronomic treatment is far less than the conventional chemotherapy (10 US dollars for metronomic versus 400 US dollars for conventional chemotherapy). In addition, there are not treatment related toxicities that warrant hospital admission, blood transfusion and the use of growth factors is also saved.

However, similar to other anticancer therapies, inherent resistance to metronomic chemotherapy is common. Moreover, even tumors that initially respond to metronomic regimens eventually develop resistance through mechanisms that are as yet unknown, it may be due to alternative angiogenic pathways; vascular co-option (enhanced ability for infiltrative tumor progression depending on the preexisting vasculature of the tumor-surrounding host tissue rather than neoangiogenesis); reduced vascular dependence (successful tumor cell adaptation to the hostile microenvironment resulting from long-term antiangiogenic therapy, characterized by oxygen, nutrient, and growth factor deprivation of the tumor cells) or rapid vascular remodeling resulting in more mature vessels, which tend to be less responsive to antiangiogenic drugs compared with newly formed immature capillaries.

In conclusion, maintenance metronomic cyclophosphamide and methotrexate demonstrated efficacy in metastatic breast cancer patients and provided durable disease stabilization especially for ER positive patients. The low costs and minimal toxicity allow patients to continue the treatment for several months and support the use of metronomic treatment as an additional therapeutic tool. More studies with larger number of patients and phase III trials are needed to confirm its significance.

\section{REFERENCES}

1. Mayer EL, Burstein HJ. Chemotherapy for metastatic breast cancer. Hematol Oncol Clin North Am 2007;21:257-72.

2. Falkson G, Gelman RS, Pandya KJ, Osborne CK, Tormey D, Cummings FJ, et al. Eastern Cooperative Oncology Group randomized trials of observation versus maintenance therapy for patients with metastatic breast cancer in complete remission following induction treatment. J Clin Oncol 1998;16:1669-76.

3. Kerbel RS. Tumor angiogenesis. N Engl J Med 2008;358:2039-49.

4. Gasparini G. Metronomic scheduling: the future of chemotherapy? Lancet Oncol 2001;2:733-40.

5. Kim KJ, Li B, Winer J, Armanini M, Gillett N, Phillips HS, et al. Inhibition of vascular endothelial growth factor-induced angiogenesis suppresses tumour growth in vivo. Nature 1993;362:841-4.

6. Browder T, Butterfield CE, Kraling BM, Shi B, Marshall B, O'Reilly MS, et al. Antiangiogenic scheduling of chemotherapy improves efficacy against experimental drug-resistant cancer. Cancer Res 2000;60:1878-86.

7. Hamano $Y$, Sugimoto $H$, Soubasakos MA, Kieran M, Olsen BR, Lawler J, et al. Thrombospondin-1 associated with tumor microenvironment contributes to low-dose cyclophosphamide-mediated endothelial cell apoptosis and tumor growth suppression. Cancer Res 2004;64:1570-4.

8. Bocci G, Nicolaou KC, Kerbel RS. Protracted low-dose effects on human endothelial cell proliferation and survival in vitro reveal a selective antiangiogenic window for various chemotherapeutic drugs. Cancer Res 2002;62:6938-43.

9. Kosmaczewska A, Ciszak L, Potoczek S, Frydecka I. The significance of Treg cells in defective tumor immunity. Arch Immunol Ther Exp (Warsz) 2008;56:181-91.

10. Lutsiak ME, Semnani RT, De Pascalis R, Kashmiri SV, Schlom J, Sabzevari $\mathrm{H}$. Inhibition of CD4(+)25+ T regulatory cell function implicated in enhanced immune response by low-dose cyclophos- 
phamide. Blood 2005;105:2862-8.

11. Colleoni M, Rocca A, Sandri MT, Zorzino L, Masci G, Nole F, et al. Low-dose oral methotrexate and cyclophosphamide in metastatic breast cancer: antitumor activity and correlation with vascular endothelial growth factor levels. Ann Oncol 2002;13:73-80.

12. Bottini A, Generali D, Brizzi MP, Fox SB, Bersiga A, Bonardi S, et al. Randomized phase II trial of letrozole and letrozole plus low-dose metronomic oral cyclophosphamide as primary systemic treatment in elderly breast cancer patients. J Clin Oncol 2006;24:3623-8.

13. Orlando L, Cardillo A, Ghisini R, Rocca A, Balduzzi A, Torrisi $R$, et al. Trastuzumab in combination with metronomic cyclophosphamide and methotrexate in patients with HER-2 positive metastatic breast cancer. BMC Cancer 2006;6:225.

14. Kerbel RS, Kamen BA. The anti-angiogenic basis of metronomic chemotherapy. Nat Rev Cancer 2004;4:423-36.

15. Dellapasqua $S$, Bertolini $F$, Bagnardi V, Campagnoli $E_{1}$ Scarano $E_{1}$ Torrisi $R$, et al. Metronomic cyclophosphamide and capecitabine combined with bevacizumab in advanced breast cancer. J Clin Oncol 2008;26:4899-905.

16. Regazzoni S, Pesce G, Marini G, Cavalli F, Goldhirsch A. Low-dose continuous intravenous infusion of 5-fluorouracil for metastatic breast cancer. Ann Oncol 1996;7:807-13.

17. Green MC, Buzdar AU, Smith T, Ibrahim NK. Weekly (wkly) paclitaxel (P) followed by FAC as primary systemic chemotherapy (PSC) of operable breast cancer improves pathologic complete remission $(\mathrm{pCR})$ rates when compared to every 3-week (0 3 wk) P therapy (tx) followed by FAC-final results of a prospective phase III randomized trial. Proc Am Soc Clin Oncol 2002;21:35A:Abstr135.

18. Nakamura J, Savinov A, Lu O, Brodie A. Estrogen regulates vascular endothelial growth/permeability factor expression in 7,12-dimethylbenz(a)anthracene-induced rat mammary tumors. Endocrinology 1996;137:5589-96.

19. Orlando L, Cardillo A, Rocca A, Balduzzi A, Ghisini R, Peruzzotti G, et al. Prolonged clinical benefit with metronomic chemotherapy in patients with metastatic breast cancer. Anticancer Drugs 2006;17: 961-7.

20. Liang Y, Brekken RA, Hyder SM. Vascular endothelial growth factor induces proliferation of breast cancer cells and inhibits the anti-proliferative activity of anti-hormones. Endocr Relat Cancer 2006;13:905-19. 